\title{
Convex hull for intersections of random lines
}

\author{
Daniel Berend $^{1}$ and Vladimir Braverman ${ }^{2 \dagger}$ \\ ${ }^{1}$ Depts. of Matematics and of Computer Science, Ben-Gurion University, Beer-Sheva 84501, Israel. berend@cs . bgu . ac. i I \\ ${ }^{2}$ Dept. of Computer Science, Ben-Gurion University, Beer-Sheva 84501, Israel. vova@cs.bgu. ac. i I
}

\begin{abstract}
The problem of finding the convex hull of the intersection points of random lines was studied in [4] and [8], and algorithms with expected linear time were found. We improve the previous results of the model in [4] by giving a universal algorithm for a wider range of distributions.
\end{abstract}

Keywords: convex hull, random lines

\section{Introduction}

Numerous problems can be reduced to finding the convex hull of a set of points - halfspace intersection, Delaunay triangulation, etc. An algorithm for finding the convex hull in the plane, known as Graham scan [5], achieves an $O(n \log n)$ running time. This algorithm is optimal in the worst case. Another algorithm [6] for the same problem runs in $O(n h)$ time, where $h$ is the number of hull points, and outperforms the preceding algorithm if $h$ happens to be very small. Kirkpatrick and Seidel [7] designed an algorithm with $O(n \log h)$ runtime, which is always at least as good as the better of the above two algorithms. A simplification of their algorithm has been recently reported by Chan [2].

For some special sets of points, it is possible to improve the results above. We concentrate on the case when the set consists of all intersection points of $n$ lines. A straightforward application of the algorithms above leads to a runtime $O\left(n^{2} \log n\right)$. Attalah [1] and Ching and Lee [3] independently presented $O(n \log n)$ runtime worst-case algorithms with $O(n)$ space. Ching and Lee [3] also showed that this result is best possible. Devroye and Toussaint [4] and Golin, Langerman and Steiger [8] studied the case when the lines are random with certain distributions. (Note that our model is different from the model of [8].) In both models, they presented algorithms with linear expected time.

Let us concentrate on the model of Devroye and Toussaint. It is convenient to use the representation of lines by points. A line not passing through the origin is uniquely determined by its intersection point with the line perpendicular to it from the origin. It is often useful to define a mechanism for selecting random lines via a mechanism for a random selection of the corresponding intersection points.

In the model of Devroye and Toussaint [4] all lines are independent identically distributed. The polar coordinates of the corresponding points are selected as follows. The distance from the origin is distributed according to some distribution law $\mathcal{R}$ (required to have a finite mean) and the angle is distributed uniformly in $[0,2 \pi)$; the distance and the angle are independent.

As mentioned earlier, their algorithm works in linear expected time. The linearity follows from a result they claim for the set of outer layer points. Here, given a set $S$, the outer layer of $S$ consists of those points $P \in S$ such that at least one quadrant around $P$ does not contain any other point of $S$. Clearly, any point in the convex hull belongs also to the outer layer. Their theorem asserts that the expected number of outer layer points is bounded above by some constant.

In fact, Devroye and Toussaint proved that, given a distribution $\mathcal{R}$, there exists a constant $C$ such that, denoting by $O_{n}$ the number of outer layer points arising from $n$ lines, we have $E\left(O_{n}\right) \leq C$ for sufficiently large $n$. If one could find a constant $C$ and an $N_{0}$ such that $E\left(O_{n}\right) \leq C$ for every $n \geq N_{0}$, independently of $\mathcal{R}$, the problem would be completely solved. However, we show by means of counter-examples that no such $C$ and $N_{0}$ exist.

As indicated above, the result of Devroye and Toussaint [4] regarding the expected number of layer points was proved under the assumption that the distribution $\mathcal{R}$ has a finite mean. Here we construct a

${ }^{\dagger}$ Partially supported by the Lynn and William Frankel Center for Computer Sciences. 
distribution with an infinite mean such that the expected number of points in the outer layer is $\Omega(n)$. Thus, their approach cannot possibly be extended to such distributions.

In this work we present another algorithm for this model. The algorithm has expected linear time, where the implied constant is global. Moreover, it works for any distribution law $\mathcal{R}$, be it of finite or of infinite mean.

In Section 2 we present the main results, and in the Sections 3,4 and 5- the proofs.

\section{The Main Results}

Given a point in the plane with polar coordinates $(r, \theta)$, denote by $L(r, \theta)$ the line passing through it and perpendicular to the line segment linking the origin with this point. If $r=0$, then the line is given by the equation $y=-(\cot \theta) x$. Let $\mathcal{R}$ be a distribution on the positive half line $\mathbf{R}_{+}=\{x \geq 0\}$, and $R_{0}, \ldots, R_{n-1}$ be independent $\mathcal{R}$-distributed random variables. Let $\Theta_{0}, \ldots, \Theta_{n-1}$ be i.i.d. random variables, uniformly distributed on $[0,2 \pi)$. For $0 \leq i \leq n-1$, denote by $L_{i}$ the line $L\left(R_{i}, \Theta_{i}\right)$. Let $y=a_{i} x+b_{i}$ be the equation of $L_{i}$. (Note that, with probability 1 , none of the $L_{i}$ 's is parallel to the $y$-axis.) Clearly, $a_{i}=-\cot \Theta_{i}$ and, with probability 1 , all $a_{i}$ 's are distinct. For each pair $(i, j), 0 \leq i<j \leq n-1$, denote by $V_{i j}$ the intersection point of the lines $L_{i}$ and $L_{j}$. Put $V(n)=\left\{V_{i j}: 0 \leq i, j \leq n-1\right\}$. Let $N_{\mathrm{ch}}^{(n)}$ be the number of points in the convex hull of $V(n)$ and $N_{\mathrm{ol}}^{(n)}$ the number of points in the outer layer of $V(n)$. Suppose the $L_{i}$ 's are sorted by their slopes. Atallah [1] showed that $C H(V(n))=C H\left(\left\{V_{i, i+1}\right.\right.$ : $i=0, \ldots, n-1\}$ ). (Here and elsewhere in the paper, the addition of indices is modulo $n$.)

As mentioned above, the reason Devroye and Toussaint's algorithm works in linear expected time is Theorem A [4, Theorem 1] If $\mathcal{R}$ has a finite mean, then there exists a universal constant $\gamma$ such that, uniformly over all $n$,

$$
E N_{\mathrm{ch}}^{(n)} \leq E N_{\mathrm{ol}}^{(n)} \leq \gamma<\infty .
$$

The constant $\gamma$ does not depend on the distribution $\mathcal{R}$.

Going over the proof of the theorem, one realizes that the authors prove the existence of a constant $\gamma$ such that, given a distribution $\mathcal{R}$, we have $E\left(O_{n}\right) \leq \gamma$ for sufficiently large $n$. The following proposition implies that, no matter how large we choose $\gamma$ to be in Theorem $A$, the initial $n$ for which 1 holds may be arbitrary large as we change $\mathcal{R}$.

Proposition 2.1 There exists a sequence of distributions $\left(\mathcal{R}_{n}\right)_{n=2}^{\infty}$ of the radius such that each $\mathcal{R}_{n}$ has mean 1 and the expected number of outer layer points in $V(n)$ under the distribution $\mathcal{R}_{n}$ exceeds $\frac{1}{2 e} n$.

What happens if the condition in Theorem A, whereby the distribution $\mathcal{R}$ has a finite expectation, is dropped? Our next result is that then Theorem A is inapplicable.

Proposition 2.2 There exists a distribution $\mathcal{R}$ with an infinite mean such that $E N_{\mathrm{ol}}^{(n)} \geq \frac{1}{2 e} n$ for each $n \geq 2$.

Note that Propositions 2.1 and 2.2 do not refer to the expected size of the convex hull, which may be much smaller than that of the outer layer. If this size is indeed uniformly bounded, the algorithm of Devroye and Toussaint [4] does work in linear expected time independently of $\mathcal{R}$. However, this does not follow from their approach.

We present here an alternative algorithm for finding the convex hull of the set of intersection points. 
Require: The input lines $L_{i}=L\left(R_{i}, \Theta_{i}\right), 0 \leq i \leq n-1$, are random and selected according to [4] (i.e., all variables are independent, the $R_{i}$ 's are $\mathcal{R}$-distributed, and the $\Theta_{i}$ 's are $U[0,2 \pi)$ ).

Ensure: $H$ is the convex hull of the set $V(n)$ of intersection points of the lines $L\left(R_{i}, \Theta_{i}\right), 0 \leq i \leq n-1$. 1: Sort the lines according to the $\theta$-coordinates of the points $\left(R_{i}, \Theta_{i}\right)$ defining them, using bucket sort.

2: For the sorted lines, find the index $k, 0 \leq k \leq n-1$, such that $\Theta_{i}<\pi$ if $i<k$. Put $\mathcal{L}_{<\pi}=\left\{L_{i}\right.$ : $i<k\}$ and $\mathcal{L}_{\geq \pi}=\left\{L_{i}: i \geq k\right\}$. /* Each of the two sets is sorted by slope.*/

3: Merge $\mathcal{L}_{\geq \pi}$ and $\mathcal{L}_{<\pi}$ into a single list, sorted by slope.

4: Calculate the intersection points of the (sorted) consecutive lines $\left\{L_{i}\right\}: V_{i}=L_{i} \cap L_{i+1}, 0 \leq i \leq$ $n-1$.

5: Let $\left(h_{i}, \psi_{i}\right)$ be the polar coordinates of $V_{i}$. Sort the $V_{i}$ 's by their $\psi$-coordinates using bucket sort.

6: Calculate $d_{i}=\psi_{i+1}-\psi_{i}, 0 \leq i<n-1$ and $d_{n-1}=\psi_{0}+2 \pi-\psi_{n-1}$ (for the newly sorted $V_{i}$ 's). Check if there exists $0 \leq k \leq n-1$ such that $d_{k} \geq \pi$.

7: if such $k$ exists then

8: $\quad$ For each $j, 0 \leq j \leq n-1$, denote by $U_{j}$ the point $V_{j+k}$ if $0 \leq j \leq n-k$ and $V_{j+k-n}$ otherwise. The set of points $U_{j}$ determines a simple polyline. Use Melkman's algorithm [9] to find $H=C H\left(\left\{U_{j}: 0 \leq j \leq n-1\right\}\right)=C H(V(n))$.

9: else

10: The origin is an internal point of $C H(V(n))$. Also, the $V_{i}$ 's are in sorted order around the origin. Use Graham's scan [11] with the origin as internal point to construct the convex hull $\mathrm{H}$.

11: end if

Algorithm 1: CONVEX HULL FOR INTERSECTIONS OF RANDOM LINES

The next theorem is our main result.

Theorem 2.3 Algorithm 1 provides a construction of the convex hull of $V(n)$, consuming linear space and expected linear time.

\section{Proofs of the Negative Results}

Proof of Proposition 2.1. Let $n \geq 3$ be arbitrarily fixed. Denote:

$$
W_{i j}=L\left(1, \Theta_{i}\right) \cap L\left(1, \Theta_{j}\right), \quad 0 \leq i<j \leq n-2 .
$$

Consider the random variable $M=\max \left\{\left\|W_{i j}\right\|: 0 \leq i<j \leq n-2\right\}$. Let $a$ be the median of $M$ : $P(M<a)=\frac{1}{2}$. Obviously, $a>1$. Denote by $\mathcal{R}_{n}$ the discrete distribution taking the two values 1 and $a$ with probabilities $1-\frac{1}{n}$ and $\frac{1}{n}$, respectively. Let $R_{0}, \ldots, R_{n-1}$ be independent $\mathcal{R}_{n}$-distributed random variables, and

$$
V_{i j}=L\left(R_{i}, \Theta_{i}\right) \cap L\left(R_{j}, \Theta_{j}\right), \quad 0 \leq i<j \leq n-1 .
$$

Consider the random variables

$$
M_{k}=\max \left\{\left\|V_{i j}\right\|: 0 \leq i<j \leq n-1, i \neq k, j \neq k\right\}, \quad k=0, \ldots, n-1
$$

Consider the events: $A=\left\{N_{\mathrm{ol}}^{(n)} \geq n-1\right\}, B_{k}=\left\{R_{k}=a, R_{j}=1 \forall j \neq k\right\}, C_{k}=\left\{M_{k}<a\right\}$ for $k=0, \ldots, n-1$. The events $B_{0} \cap C_{0}, \ldots, B_{n-1} \cap C_{n-1}$ are equi-probable and pairwise disjoint. If $B_{k} \cap C_{k}$ occurs for some $k$, then all points $V_{i j}, i, j \neq k$, are at a distance smaller than $a$ from the origin, while the points $V_{i j}, i \neq k$, are at a distance at least $a$. Hence in this case all points $V_{i k}, i \neq k$, are outer layer points; see Figure 1. (In fact, some of the $L_{i}$ 's may be parallel to $L_{k}$, in which case we miss the corresponding intersection points, but the probability for this is 0 .) Hence $\bigcup_{k=0}^{n-1}\left(B_{k} \cap C_{k}\right) \subseteq A$.

Hence:

$$
P(A) \geq P\left(\bigcup_{k=0}^{n-1}\left(B_{k} \cap C_{k}\right)\right)=n P\left(B_{0} \cap C_{0}\right)=n P\left(B_{0}\right) P\left(C_{0} \mid B_{0}\right) .
$$

Further we obtain

$$
\begin{aligned}
P\left(C_{0} \mid B_{0}\right) & =P\left(M_{0}<a \mid R_{0}=a, R_{1}=1, \ldots, R_{n-1}=1\right) \\
& =P\left(M_{0}<a \mid R_{1}=1, \ldots, R_{n-1}=1\right)=P(M<a)=\frac{1}{2} .
\end{aligned}
$$




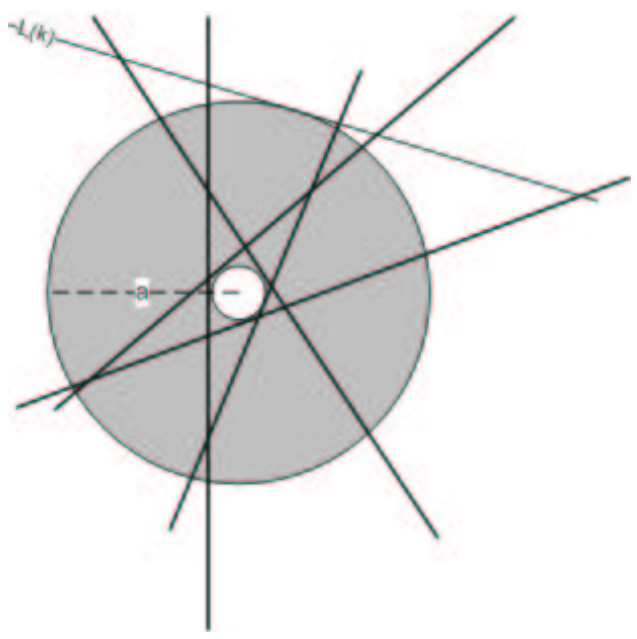

Fig. 1: Outer layer points

Therefore,

$$
P(A) \geq n \frac{1}{n}\left(1-\frac{1}{n}\right)^{n-1} \frac{1}{2}>\frac{1}{2 e} .
$$

Consequently,

$$
E N_{\mathrm{ol}}^{(n)} \geq P(A) \cdot(n-1)+(1-P(A)) \cdot 1 \geq \frac{1}{2 e} n .
$$

Since for $n=2$ we have $E N_{\mathrm{ol}}^{(2)}=1$, the inequality holds in this case as well.

\section{Proof of Proposition 2.2}

Define a sequence $\left(a_{m}\right)_{m=1}^{\infty}$ inductively. Put $a_{1}=1$. Let $m \geq 1$ be arbitrary and fixed, and suppose $a_{1}, a_{2}, \ldots, a_{m}$ have been defined. Let $\left(\Theta_{i}\right)_{i=0}^{m-1}$ be independent $U[0,2 \pi)$-distributed random variables. Denote

$$
\begin{aligned}
& W_{i j}^{s t}=L\left(a_{s}, \Theta_{i}\right) \cap L\left(a_{t}, \Theta_{j}\right), \quad 1 \leq s, t \leq m, 0 \leq i<j \leq m-1, \\
& M_{m}=\max \left\{\left\|W_{i j}^{s t}\right\|: \quad 1 \leq s, t \leq m, 0 \leq i<j \leq m-1\right\} .
\end{aligned}
$$

Let $b_{m+1}$ be the median of $M_{m}$ such that $P\left(M_{m}<b_{m+1}\right)=\frac{1}{2}$, and put $a_{m+1}=\max \left(b_{m+1}, m\right)$. Define a distribution $\mathcal{R}$ by: $R \sim \mathcal{R}$ if

$$
P\left(R=a_{m}\right)=\frac{1}{m(m+1)}, \quad m=1,2, \ldots
$$

Clearly, $E(R)=\infty$. We shall prove that $\mathcal{R}$ satisfies the required condition. Let $R_{0}, \ldots, R_{n-1}$ be independent $\mathcal{R}$-distributed random variables, and denote

$$
\begin{aligned}
& V_{i j}=L\left(R_{i}, \Theta_{i}\right) \cap L\left(R_{j}, \Theta_{j}\right), \quad 0 \leq i<j \leq n-1, \\
& H_{k}=\max \left\{\left\|V_{i j}\right\|: 0 \leq i<j \leq n-1, i \neq k, j \neq k\right\}, \quad k=0, \ldots, n-1 .
\end{aligned}
$$

Consider the events $A=\left\{N_{\mathrm{ol}}^{(n)} \geq n-1\right\}, \quad B_{k}=\left\{R_{k} \geq a_{n}, R_{j} \leq a_{n-1} \forall j \neq k\right\}, \quad C_{k}=\left\{H_{k}<a_{n}\right\}$ for $k=0, \ldots, n-1$. According to the definition of $\mathcal{R}$,

$$
P\left(R_{0} \geq a_{n}\right)=\sum_{m=n}^{\infty} \frac{1}{m(m+1)}=\frac{1}{n},
$$

which implies

$$
P\left(B_{0}\right)=\frac{1}{n}\left(1-\frac{1}{n}\right)^{n-1}
$$


The events $B_{0} \cap C_{0}, \ldots, B_{n-1} \cap C_{n-1}$ are equi-probable and pairwise disjoint and $\bigcup_{k=0}^{n-1}\left(B_{k} \cap C_{k}\right) \subseteq A$. Hence

$$
P(A) \geq P\left(\bigcup_{k=0}^{n-1}\left(B_{k} \cap C_{k}\right)\right)=n P\left(B_{0} \cap C_{0}\right)=n P\left(B_{0}\right) P\left(C_{0} \mid B_{0}\right) .
$$

Further, we obtain

$$
\begin{aligned}
P\left(C_{0} \mid B_{0}\right) & =P\left(H_{0}<a_{n} \mid R_{0} \geq a_{n}, R_{1}<a_{n}, \ldots, R_{n-1}<a_{n}\right) \\
& =P\left(H_{0}<a_{n} \mid R_{1}<a_{n}, \ldots, R_{n-1}<a_{n}\right) \geq P\left(M_{n-1}<a_{n}\right) \geq \frac{1}{2} .
\end{aligned}
$$

Therefore,

$$
P(A) \geq n \cdot \frac{1}{n}\left(1-\frac{1}{n}\right)^{n-1} \cdot \frac{1}{2}>\frac{1}{2 e} .
$$

Consequently, $E N_{\mathrm{ol}}^{(n)} \geq P(A) \cdot(n-1)+(1-P(A)) \cdot 1 \geq \frac{1}{2 e} n$.

\section{Cyclic Functions}

Our work depends on numerous computations involving angles. It will be convenient to view angles as points of the circle group $\mathbb{T}=\mathbb{R} / 2 \pi \mathbb{Z}$. As representatives of the cosets we shall take points in $[0,2 \pi)$ (with the quotient topology of $\mathbb{R} / 2 \pi \mathbb{Z}$ ). Addition modulo $2 \pi$ will be denoted by $\oplus$ and subtraction by $\ominus$. Let $X_{0}, \ldots, X_{n-1} \sim U[0,2 \pi)$ be independent random variables.

Definition 4.1 A function $f: \mathbb{T} \times \mathbb{T} \mapsto \mathbb{T}$ is cyclic if $f(x \oplus \alpha, y \oplus \alpha)=f(x, y) \oplus \alpha, \quad x, y, \alpha \in \mathbb{T}$.

Let $\rho$ be the random permutation of $\{0 \ldots n-1\}$ which determines the index of the successor of each $X_{i}$ when the values are ordered on $\mathbb{T}$. More accurately, $\rho(i), i=0, \ldots, n-1$, is the index $j, 0 \leq j \leq n-1$, such that $X_{j}$ is the nearest point to $X_{i}$ on the unit circle (in the counterclockwise direction):

$$
X_{\rho(i)} \ominus X_{i}=\min _{0 \leq k \leq n-1, k \neq i}\left(X_{k} \ominus X_{i}\right)
$$

In the following two lemmas, we shall deal with functions $f: \mathbb{T}^{2} \times \mathbb{R}^{2} \mapsto \mathbb{T}$ whose restriction $f\left(\cdot, \cdot, r_{1}, r_{2}\right)$ is cyclic for arbitrary fixed $r_{1}, r_{2}$. By $R_{0}, \ldots, R_{n-1}$ we shall denote i.i.d. random variables independent of $X_{0}, \ldots, X_{n-1}$.

Denote $I=\left[0, \frac{2 \pi}{n}\right)$ and $M=\sum_{i=0}^{n-1} \mathbf{1}_{I}\left(f\left(X_{i}, X_{\rho(i)}, R_{i}, R_{\rho(i)}\right)\right)$. The proofs of the next two lemmas are omitted due to their length. They can be found in the full version of the paper.

Lemma 4.2 $E(M)=1$.

Lemma 4.3 $E\left(M^{2}\right) \leq 3+\frac{n(n-1)}{(n-2)(n-3)}$.

\section{Proof of the Main Result}

This section is devoted to the proof of Theorem 2.3. For a pair of points $\theta, \eta \in[0,2 \pi)$, let $A(\theta, \eta)$ be the arc from $\theta$ to $\eta$, taken counterclockwise:

$$
A(\theta, \eta)=\{\nu \in[0,2 \pi):(\eta \ominus \nu)+(\nu \ominus \theta)=\eta \ominus \theta\} .
$$

The following lemmas describe some properties of $A$. The proofs are simple, and will be omitted.

Lemma 5.1 Let $\alpha, \beta, \gamma, \eta, \theta \in \mathbb{T}$ be points such that $\eta \in A(\alpha, \beta), \theta \in A(\beta, \gamma)$ and $\beta \in A(\alpha, \gamma)$. Then $\beta \in A(\eta, \theta)$.

Lemma 5.2 Let $\alpha, \beta, \gamma, \eta, \theta, \zeta$ be points such that $\theta \in A(\alpha, \beta), \zeta \in A(\gamma, \eta), \beta \in A(\alpha, \gamma)$ and the two sets $A(\alpha, \beta)$ and $A(\gamma, \eta)$ intersect in at most the single point $\gamma$. Then $A(\beta, \gamma) \subseteq A(\theta, \zeta) \subseteq A(\alpha, \eta)$. 
Lemma 5.3 Let $\alpha, \beta, \gamma, \eta$ be points such that $\beta \ominus \alpha<\pi$ and $\eta, \gamma \in A(\alpha, \beta)$. Then $A(\gamma, \eta) \subseteq A(\alpha, \beta)$ if and only if $\eta \ominus \gamma<\pi$.

For $0 \leq \theta<2 \pi$, denote $\underline{\theta}=\max \{\theta, \theta \oplus \pi\}$ and $\bar{\theta}=\min \{\theta, \theta \oplus \pi\}$. Note that $0 \leq \bar{\theta}<\pi \leq \underline{\theta}=$ $\bar{\theta}+\pi<2 \pi$. Thus, $\bar{\theta}$ is always at the top half circle and $\underline{\theta}$ at the bottom half circle (which is the reason for our notation).

Lemma 5.4 Let $\alpha, \beta$ be angles such that $-\cot \alpha<-\cot \beta$. Then $\bar{\alpha}<\bar{\beta}, \underline{\alpha}<\underline{\beta}$ and $\underline{\beta} \ominus \bar{\alpha}>\pi$.

Lemma 5.5 Let $l=L(r, \theta), l^{\prime}=L\left(r^{\prime}, \theta^{\prime}\right)$ be two intersecting lines. Denote by $(\psi, h)$ the polar representation of the intersection point $l \cap l^{\prime}$. If $\theta^{\prime} \ominus \theta>\pi$, then $\psi \in A\left(\theta \ominus \frac{\pi}{2}, \theta^{\prime} \oplus \frac{\pi}{2}\right)$.

Lemma 5.6 Let $r_{1}, r_{2} \geq 0$ be two real numbers and $\theta_{1}, \theta_{2}$ be two angles such that $-\cot \theta_{1}<-\cot \theta_{2}$. Denote $\hat{V}=L\left(r_{1}, \bar{\theta}_{1}\right) \cap L\left(r_{2}, \underline{\theta}_{2}\right)$. Let $(\hat{r}, \hat{\theta})$ be the polar representation of $\hat{V}$. Then $\hat{\theta} \in A\left(\underline{\theta}_{1} \oplus \frac{\pi}{2}, \underline{\theta}_{2} \oplus \frac{\pi}{2}\right)$.

Proof Due to Lemma 5.4, $\underline{\theta}_{2} \ominus \bar{\theta}_{1}>\pi$. Using Lemma 5.5 and the fact that $\bar{\theta} \ominus \frac{\pi}{2}=\underline{\theta} \oplus \frac{\pi}{2}$ for any $\theta \in[0,2 \pi)$, we have

$$
\hat{\theta} \in A\left(\bar{\theta}_{1} \ominus \frac{\pi}{2}, \underline{\theta}_{2} \oplus \frac{\pi}{2}\right)=A\left(\underline{\theta}_{1} \oplus \frac{\pi}{2}, \underline{\theta}_{2} \oplus \frac{\pi}{2}\right) .
$$

Lemma 5.7 Let $J=A(\theta, \eta)$ be an arc such that $\eta \ominus \theta<\pi$. Let $\alpha, \beta, \gamma, \zeta \in \mathbb{T}$ be angles such that $-\cot \alpha<-\cot \beta<-\cot \gamma$ and $\underline{\alpha} \oplus \zeta, \underline{\gamma} \oplus \zeta \in J$. Then $\underline{\beta} \oplus \zeta \in J$.

Proof of Lemma 5.7 Using Lemma 5.4, we obtain $\underline{\gamma} \ominus \underline{\alpha}<\pi$. Therefore we can apply Lemma 5.3 to conclude that $A(\underline{\alpha} \oplus \zeta, \underline{\gamma} \oplus \zeta) \subseteq J$. According to Lemma 5.4, $\underline{\alpha}<\underline{\beta}<\underline{\gamma}$ and $\underline{\alpha}, \underline{\beta}, \underline{\gamma} \in[\pi, 2 \pi)$. Thus $\underline{\beta} \in A(\underline{\alpha}, \underline{\gamma})$ and $\underline{\beta} \oplus \zeta \bar{\epsilon} A(\underline{\alpha} \oplus \zeta, \underline{\gamma} \oplus \zeta) \subseteq J$.

We now turn to prove Theorem 2.3. To this end, we have to deal with various stages of Algorithm 1. Recall that, after step 4 of Algorithm 1, the lines $L_{i}=L\left(R_{i}, \Theta_{i}\right), 0 \leq i \leq n-1$, are sorted by slope. Denote

$$
\hat{V}_{i}=L\left(R_{i}, \bar{\Theta}_{i}\right) \cap L\left(R_{i+1}, \underline{\Theta}_{i+1}\right), \quad 0 \leq i \leq n-1,
$$

and let $\hat{\psi}_{i}$ be the angle between the positive $x$-axis and the line segment connecting 0 with $\hat{V}_{i}$.

Lemma 5.8 Let $J=A(\tau, \sigma)$ be an arc such that $\sigma \ominus \tau<\pi$. For any $n$

$$
\sum_{i=0}^{n-1} \mathbf{1}_{J}\left(\hat{\psi}_{i}\right) \leq \sum_{i=0}^{n-1} \mathbf{1}_{J}\left(\underline{\Theta}_{i} \oplus \frac{\pi}{2}\right)+2 .
$$

Proof If the set $\left\{i: \hat{\psi}_{i} \in J\right\}$ contains at most two elements, then the required inequality is trivial. Suppose that $\left|\left\{i: \hat{\psi}_{i} \in J\right\}\right|>2$. Denote $l=\min _{0 \leq i \leq n-2}\left\{i: \hat{\psi}_{i} \in J\right\}, k=\max _{0 \leq i \leq n-2}\left\{i: \hat{\psi}_{i} \in J\right\}$. Putting $\hat{V}_{l}=\left(\hat{\psi}_{l}, \hat{r}_{l}\right)$ and applying Lemma 5.6 with $\theta_{1}=\Theta_{l}, \theta_{2}=\Theta_{l+1}, \hat{V}=\hat{V}_{l}, r_{1}=R_{l}, r_{2}=R_{l+1}$, we conclude that $\hat{\psi}_{l} \in A\left(\underline{\Theta}_{l} \oplus \frac{\pi}{2}, \underline{\Theta}_{l+1} \oplus \frac{\pi}{2}\right)$.

Similarly, applying Lemma 5.6 with $\theta_{1}=\Theta_{k}, \theta_{2}=\Theta_{k+1}, \hat{V}=\hat{V}_{k}, \quad r_{1}=R_{l}, \quad r_{2}=R_{l+1}$, we conclude that $\hat{\psi}_{k} \in A\left(\underline{\Theta}_{k} \oplus \frac{\pi}{2}, \underline{\Theta}_{k+1} \oplus \frac{\pi}{2}\right)$.

Since $\underline{\Theta}_{l} \leq \underline{\Theta}_{l+1} \leq \underline{\Theta}_{k}$, we have $\underline{\Theta}_{l+1} \oplus \frac{\pi}{2} \in A\left(\underline{\Theta}_{l} \oplus \frac{\pi}{2}, \underline{\Theta}_{k} \oplus \frac{\pi}{2}\right)$. Applying Lemma 5.2 with

$$
\alpha=\underline{\Theta}_{l} \oplus \frac{\pi}{2}, \beta=\underline{\Theta}_{l+1} \oplus \frac{\pi}{2}, \gamma=\underline{\Theta}_{k} \oplus \frac{\pi}{2}, \eta=\underline{\Theta}_{k+1} \oplus \frac{\pi}{2}, \theta=\hat{\psi}_{l}, \zeta=\hat{\psi}_{k},
$$

we obtain

$$
\underline{\Theta}_{l+1} \oplus \frac{\pi}{2}, \underline{\Theta}_{k} \oplus \frac{\pi}{2} \in A\left(\hat{\psi}_{l}, \hat{\psi}_{k}\right)
$$

and

$$
\hat{\psi}_{k} \ominus \hat{\psi}_{l}=\zeta \ominus \theta \leq \eta \ominus \alpha=\left(\underline{\Theta}_{k+1} \oplus \frac{\pi}{2}\right) \ominus\left(\underline{\Theta}_{l} \oplus \frac{\pi}{2}\right) .
$$

Thus $\hat{\psi}_{k} \ominus \hat{\psi}_{l} \leq \underline{\Theta}_{k+1} \ominus \underline{\Theta}_{l} \leq \pi$. Applying Lemma 5.3 with $\alpha=\tau, \beta=\sigma, \gamma=\hat{\psi}_{l}, \eta=\hat{\psi}_{k}$, we get:

$$
A\left(\hat{\psi}_{l}, \hat{\psi}_{k}\right)=A(\gamma, \eta) \subseteq A(\alpha, \beta)=A(\tau, \sigma)=J .
$$

Taking into account that

$$
\underline{\Theta}_{l+1} \oplus \frac{\pi}{2}, \underline{\Theta}_{k} \oplus \frac{\pi}{2} \in A\left(\hat{\psi}_{l}, \hat{\psi}_{k}\right)
$$


we therefore have

$$
\underline{\Theta}_{l+1} \oplus \frac{\pi}{2}, \underline{\Theta}_{k} \oplus \frac{\pi}{2} \in J .
$$

By Lemma 5.7, for any $l+1 \leq h \leq k$ we have $\underline{\Theta}_{h} \oplus \frac{\pi}{2} \in J$. Finally,

$$
\sum_{i=0}^{n-1} \mathbf{1}_{J}\left(\hat{\psi}_{i}\right) \leq \sum_{i=0}^{n-2} \mathbf{1}_{J}\left(\hat{\psi}_{i}\right)+1 \leq k-l+2 \leq \sum_{i=0}^{n-1} \mathbf{1}_{J}\left(\underline{\Theta}_{i} \oplus \frac{\pi}{2}\right)+2 .
$$

Similarly, let $\check{V}_{i}=L\left(R_{i}, \underline{\Theta}_{i}\right) \cap L\left(R_{i+1}, \bar{\Theta}_{i+1}\right)$. Let $\left(\check{r}_{i}, \check{\psi}_{i}\right)$ be the polar representation of $\check{V}_{i}$. Using the same observations as in Lemma 5.8, we obtain

Lemma 5.9 Let $J=A(\tau, \sigma)$ be an arc such that $\sigma \ominus \tau<\pi$. For any $n$

$$
\sum_{i=0}^{n-1} \mathbf{1}_{J}\left(\check{\psi}_{i}\right) \leq \sum_{i=0}^{n-1} \mathbf{1}_{J}\left(\bar{\Theta}_{i} \oplus \frac{\pi}{2}\right)+2 .
$$

We shall refer to $V_{i}$ as "far" if $\Theta_{i+1} \ominus \Theta_{i}>\pi$ and as "near" otherwise. Let $F$ be the set of indices $i, 0 \leq i \leq n-1$, for which $V_{i}$ is far, and $N$ the set of those for which it is near. Put $I=\left[0, \frac{2 \pi}{n}\right)$.

Lemma 5.10 For any $n$ :

$$
E\left(\sum_{i \in F} \mathbf{1}_{I}\left(\psi_{i}\right)\right)^{2}<40 .
$$

Proof Clearly, if $V_{i}$ is a "far" point, then $V_{i} \in\left\{\hat{V}_{i}, \check{V}_{i}\right\}$, which implies

$$
E\left(\sum_{i \in F} \mathbf{1}_{I}\left(\psi_{i}\right)\right)^{2} \leq E\left(\sum_{i=1}^{n} \mathbf{1}_{I}\left(\hat{\psi}_{i}\right)+\mathbf{1}_{I}\left(\check{\psi}_{i}\right)\right)^{2}
$$

Using Lemmas 5.8, 5.9 and the fact that $\underline{\Theta}_{i} \in\left\{\Theta_{i}, \Theta_{i}+\pi\right\}$, we obtain that

$$
E\left(\sum_{i=1}^{n} \mathbf{1}_{I}\left(\hat{\psi}_{i}\right)+\mathbf{1}_{I}\left(\check{\psi}_{i}\right)\right) \leq \sum_{i=0}^{n-1} \mathbf{1}_{I}\left(\Theta_{i} \oplus \frac{\pi}{2}\right)+\sum_{i=0}^{n-1} \mathbf{1}_{I}\left(\Theta_{i} \oplus \frac{3 \pi}{2}\right)+4
$$

Therefore,

$$
\begin{aligned}
E\left(\sum_{i \in F} \mathbf{1}_{I}\left(\psi_{i}\right)\right)^{2} \leq & E\left(\sum_{i=0}^{n-1} \mathbf{1}_{I}\left(\Theta_{i} \oplus \frac{\pi}{2}\right)+\sum_{i=0}^{n-1} \mathbf{1}_{I}\left(\Theta_{i} \oplus \frac{3 \pi}{2}\right)\right)^{2} \\
& +16 E\left(\sum_{i=0}^{n-1} \mathbf{1}_{I}\left(\Theta_{i} \oplus \frac{\pi}{2}\right)\right)+16
\end{aligned}
$$

Now, $\sum_{i=0}^{n-1} \mathbf{1}_{I}\left(\Theta_{i} \oplus \frac{\pi}{2}\right)$ and $\sum_{i=0}^{n-1} \mathbf{1}_{I}\left(\Theta_{i} \oplus \frac{3 \pi}{2}\right)$ are both $B\left(n, \frac{1}{n}\right)$-distributed, and hence

$$
E\left(\sum_{i=0}^{n-1} \mathbf{1}_{I}\left(\hat{\psi}_{i}\right)\right)^{2} \leq 2 E\left(\sum_{i=0}^{n-1} \mathbf{1}_{I}\left(\Theta_{i} \oplus \frac{\pi}{2}\right)\right)^{2}+2 E\left(\sum_{i=0}^{n-1} \mathbf{1}_{I}\left(\Theta_{i} \oplus \frac{3 \pi}{2}\right)\right)^{2}+32 \leq 40 .
$$

Lemma 5.11 Let $\alpha, \beta$ be angles such that $\beta \ominus \alpha \leq \pi$ and $-\cot \alpha<-\cot \beta$. Then $\alpha$ and $\beta$ belong to the same semicircle i.e., either $\alpha, \beta \in[0, \pi)$ or $\alpha, \beta \in[\pi, 2 \pi)$.

Proof Suppose, say, that $\alpha \in[0, \pi)$ and $\beta \in[\pi, 2 \pi)$. Then $\alpha+\pi \in[\pi, 2 \pi)$ and $\alpha+\pi \geq \beta$. Therefore $-\cot \alpha=-\cot (\alpha+\pi) \geq-\cot \beta$, which contradicts the conditions.

Let $\rho$ be the permutation of $\{0,1, \ldots, n-1\}$ such that $\Theta_{\rho(i)}$ is the nearest to $\Theta_{i}$ in the counterclockwise direction among all $\Theta_{k}$ 's:

$$
\Theta_{\rho(i)} \ominus \Theta_{i}=\min _{0 \leq k \leq n-1, k \neq i}\left(\Theta_{k} \ominus \Theta_{i}\right), \quad i=0,1, \ldots, n-1 .
$$

Lemma 5.12 For all $i \in N$ we have $\rho(i)=i+1$. 
Proof We may assume that $\Theta_{i} \neq \Theta_{i+1}$, for any $0 \leq i \leq n-1$. . According to Lemma 5.11, the angles $\Theta_{i}, \Theta_{i+1}$ belong to the same semicircle. The function - cot is increasing on $(0, \pi)$ and $(\pi, 2 \pi)$, therefore $\Theta_{i+1}>\Theta_{i}$. Suppose that there exists $0 \leq k \leq n-1$ such that $\Theta_{i}<\Theta_{k}<\Theta_{i+1}$. It is easy to see that $\Theta_{k}$ belongs to the same semicircle as $\Theta_{i}, \Theta_{i+1}$, and therefore $a_{i}<a_{k}<a_{i+1}$. This contradicts the increasing order of the slopes $a_{i}$ of $L_{i}$ 's.

Lemma 5.13E $\left(\sum_{i \in N} \mathbf{1}_{I}\left(\psi_{i}\right)\right)^{2}<3+\frac{n(n-1)}{(n-2)(n-3)}$.

Proof Let $\left(\psi_{i}^{\prime}, r_{i}^{\prime}\right)$ be the polar representation of the point $V_{i, \rho(i)}$. We have, according to Lemma 5.12:

$$
\sum_{i \in N} \mathbf{1}_{I}\left(\psi_{i}\right) \leq \sum_{i=0}^{n-1} \mathbf{1}_{I}\left(\psi_{i}^{\prime}\right)
$$

It is easy to verify that

$$
\psi_{i}^{\prime}=\arctan \left(\frac{R_{i} \cos \Theta_{\rho(i)}-R_{\rho(i)} \cos \Theta_{i}}{R_{\rho(i)} \sin \Theta_{i}-R_{i} \sin \Theta_{\rho(i)}}\right) .
$$

Denote

$$
f\left(\theta_{1}, \theta_{2}, r_{1}, r_{2}\right)=\arctan \left(\frac{r_{1} \cos \theta_{2}-r_{2} \cos \theta_{1}}{r_{2} \sin \theta_{1}-r_{1} \sin \theta_{2}}\right) .
$$

The function is cyclic according to Definition 4.1. Therefore, by Lemma 4.3:

$$
E\left(\sum_{i=0}^{n-1} \mathbf{1}_{I}\left(\psi_{i}^{\prime}\right)\right)^{2}=E\left(\sum_{i=0}^{n-1} \mathbf{1}_{I}\left(f\left(\Theta_{i}, \Theta_{\rho(i)}, R_{i}, R_{\rho(i)}\right)\right)\right)^{2} \leq 3+\frac{n(n-1)}{(n-2)(n-3)} .
$$

Lemma $5.14 E\left(\sum_{i=0}^{n-1} \mathbf{1}_{I}\left(\psi_{i}\right)\right)^{2} \leq 86+\frac{n(n-1)}{(n-2)(n-3)}$.

Proof According to lemmas 5.10, 5.13,

$$
E\left(\sum_{i=0}^{n-1} \mathbf{1}_{I}\left(\psi_{i}\right)\right)^{2} \leq 2 E\left(\sum_{i \in F} \mathbf{1}_{I}\left(\psi_{i}\right)\right)^{2}+2 E\left(\sum_{i \in N} \mathbf{1}_{I}\left(\psi_{i}\right)\right)^{2} \leq 86+\frac{2 n(n-1)}{(n-2)(n-3)} .
$$

Lemma 5.15 Step 5 of algorithm 1 sorts the $\psi_{i}$ 's in expected linear time.

Proof To show that bucket sort works in expected linear time on the $\psi_{i}$ 's, it is sufficient to show that there exists a constant $\mathrm{C}$, such that $E\left(\sum_{i=0}^{n-1} \mathbf{1}_{I}\left(\psi_{i}\right)\right)^{2}<C$. According to Lemma 5.14, this is in fact true.

Proof of Theorem 2.3

The algorithm indeed calculates the required convex hull due to [1], [9] and [10]. The algorithm works in expected linear time due to Lemma 5.15 .

\section{References}

[1] M. Atallah. Computing the convex hull of line intersections. J. Algorithms, 7:285-288, 1986.

[2] T.M. Chan. Optimal output-sensitive convex hull algorithms in two and three dimensions. Discrete \& Computational Geometry, 16:361-368, 1996.

[3] Y.T. Ching and D.T. Lee. Finding the diameter of a set of lines. Pattern Recognition, 18:249-255, 1985.

[4] L. Devroye and G. Toussaint. Convex hulls for random lines. J. Algorithms, 14:381-394, 1993.

[5] R.L Graham. An efficient algorithm for determining the convex hull of a finite planar set. Information Processing Letters, 1:132-133, 1972. 
[6] R.A. Jarvis. On the identification of the convex hull of a finite set of points in the plane. Information Processing Letters, 2:18-21, 1973.

[7] D.G Kirkpatrick and R. Seidel. The ultimate planar convex hull algorithm? SIAM J. Comput., 15:287-299, 1986.

[8] S. Langerman M. Golin and W. Steiger. The convex hull for random lines in the plane. In Proceedings of the Japan Conference on Discrete and Computational Geometry (JCDCG 2002), 2002.

[9] A.A. Melkman. On-line construction of the convex hull of a simple polyline. Information Processing Letters, 25:11-12, 1987.

[10] F.P. Preparata and M.I. Shamos. Computational Geometry: An Introduction. Springer-Verlag, NewYork, 1986.

[11] G.E. Leiserson T.H. Cormen and R.L. Rivest. Introduction to Algorithms. The MIT Press, 2000. 
